\title{
Simplification of sound-pressure characteristics using narrow flow channels subject with temperature gradient
}

\author{
Tomoaki Kyoden ${ }^{1 *}$ \\ ${ }^{1}$ National institute of Technology, Toyama College, Imizu, Toyama, Japan
}

\begin{abstract}
A thermoacoustic engine is one of promising system for utilization of unused energy. The object of this paper is to study the system of unused energy, such as heat energy and sound energy in noise using thermoacoustic engine. The thermoacoustic engine is composed of a stack sandwiched by two heat exchangers and tube. The stack has many narrow flow channels and its length for $50 \mathrm{~mm}$. For example, in order to create narrow flow channels, the stack is comprised of wire-mesh screen. When the steep temperature gradient is made in the stack, a self-exited gas oscillation is generated. Thus, heat energy can be converted into sound energy. In this paper, thermoacoustic conversion device applied for noise energy generated from engine plants was presented. The noise has complicated frequency characteristics and low energy density. We demonstrated selective amplification of acoustic pressure only one-frequency in noise by utilization of the technique of thermoacoustic engine. In addition, the other frequencies were attenuated by viscosity dissipation in the stack. Summarizing these results, the simplification and amplified sound was created from complex noise.
\end{abstract}

\section{Introduction}

In order to realizing sustainable growth in a low-carbon society, utilization of unused energy is an important issue. The energy conversion techniques utilizing thermoacoustic phenomena, which are based on heat transport using compression and expansion of gas in many narrow flow channel called stack, is one of promising method, because of its availability for various energy sources, simple structure with non-moving parts and no harmful gas for refrigeration. The thermoacoustic energy conversion system have been propsed into two types: one is thermoacoustic engine [1-6], and other is thermoacoustic cooler. These thermoacousitc engine and cooler have been studied actively.

In the case of thermoacoustic engine, self-excited oscillation in pipe is caused by installing the stack which has steep temperature gradient $[7,8]$. The heat energy can be converted into sound energy due to the thermoacoustic effect. On the other hand, when the sound wave passes through the narrow flow channel, the heat transport due to compression and expansion of gas occurs. Thus, heat pump action is induced by sound wave. Thermoacoustic phenomena are attractive effect for development of new type of energy conversion devise.. Moreover, acoustic amplification by thermoacoustic effect was proposed by Ceperley [9], and it was realized by Biwa et al. $[10,11]$. These recent surge of research activity had brought significant findings. However, most of these research paid attention to waste heat or solar energy as input source energy. In order to extend the application of the thermoacoustic device, development of the system for various unused energy is important.

We propose a new energy harvesting technology using thermoacoustic phenomena. Specifically, by using a thermoacoustic phenomenon in which acoustic waves are induced by providing a rapid temperature gradient (about $200^{\circ} \mathrm{C}$. to about $500^{\circ} \mathrm{C}$.) in a fine flow path, noise of low energy density can be selectively The final goal is to propose a system that amplifies and leads to effective utilization. In this paper, we carried out the basic study of connecting method between thermoacoustic field and a noise. Especially, sound pressure amplified effect. The effect of thermoacoustic pressure amplification was measured experimentally. Summarizing this result, the effect of thermoacoustic amplification was discussed in relation to the stack structure and resonance state.

\section{Experimental apparatus}

Figure 1 illustrates a schematic diagram of the experimental apparatus. The resonance pipe was a circular pipe of 1,69 $\mathrm{m}$ in length. Its inner diameter was for $24 \mathrm{~mm}$. The end of pipe was closed and the the speaker was set at the other end. The atmospheric air was filled in pipe. A sound wave was injected from the speaker. The coordinate $X$ was defined along axis of the passage, and origin of $X$ was set at the end of pipe. The pressure transducers were set on the wall and closed end. The thermoacoustic section was consisted of a stack at $X$ $=507 \sim 557 \mathrm{~mm}$ and two heat exchangers (these thickness was for 10-mm). The top of the stack was set

\footnotetext{
Corresponding author: kyouden@nc-toyama.ac.jp
} 
at one-third length of the pipe. The one of heat exchanger was heated by heater. And the other was kept at room temperature. The temperature difference between top and bottom of the stack was kept to $300 \mathrm{~K}$. When the sound wave passes through the stack, the sound energy increased by thermoacoustic effect.

In this study, the stack was composed of stainless-steel mesh screens. In the experiments, sound with selected frequency was supplied by using speaker driven by electric input power of $1 \mathrm{~W}$.

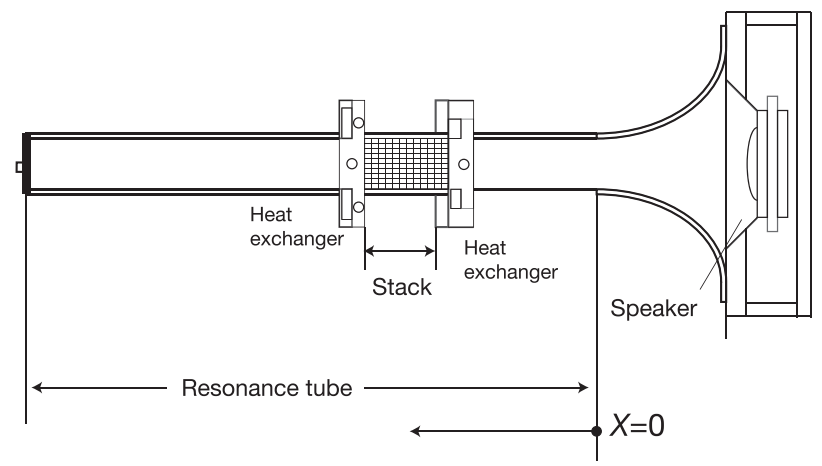

Fig. 1. Experimental apparatus.

\section{Results and Discussion}

\subsection{Resonans state of circuler pipe with closed end}

Firstly, we mesured the sound pressue at closed end for cralyfing resonance state. The resonance frequency are defined at maximum value of pressure. The consumption power of speaker was comstant for $1 \mathrm{~W}$. Fig. 2 shows the resonance curves of pressure at closed end $(X=1.69$ $\mathrm{m})$. White circles were results without stack, and red circles were with stack and temperature gradient $(300 \mathrm{~K})$, In the circular pipe with closed end, sound resonance with odd number times of a one-quarter wavelength is occurred, which is called the first harmonic, third harmonic, and fifth harmonic mode and more. In this paper, since the length of circular pipe $L$ is for $1.69 \mathrm{~m}$, the third harmonics and fifth harmonics mode were obtained when maximum pressure at the 50, 150 and 248 $\mathrm{Hz}$, respectively. By comparing with case without stack and case with stack, it was found that the sound was attenuated by installing the stack, especially fifth harmonic frequency at 50 and $208 \mathrm{~Hz}$. The number of stack which was used for stack was \#30 and \#60.

\subsection{Applied to noise as sound source}

In general noise has complex frequency characteristics. The noise energy could be simplified by using resonce effect and thermoacoustic device. Therefore, we choised the engine room noise in WAKASHIMARU. WAKASHIOMARU is trainning ship of
Toyamanational institute of Technology, Toyama College.

Figure 2 shows the comparing between sound frequency characteristics, one is sound noise in the engine room in WAKASHIMARU and other one is measurement pressure amplitude at closed end under using the noise as sound sourse. In the case of the engine room noise, the dominant frequencies were spread out under $1 \mathrm{kHz}$. These frequencies were generated by main engine. Main engine is largest system in the engine room. The low frequency is generated large system. And higher value of frequencies were generated from dynamo generator. The dynamo generator has higher rotation speed comparing to the main engine.

The engine room noise was captured in a computer. By using the noise data in the computer, the engine room noise could be outputted from speaker using function generator. Therefore, we could use the engine room noise as sound source. The sound pressure measured at closed end is shown in Fig. 3 using red line. The highest value of pressure frequency was at $150 \mathrm{~Hz}$. This frequency was third harmonic frequency in the experimental device. In addition, any frequencies were not appeared, especially high frequencies' region. These frequencies were difference at resonance frequencies, and the resonance frequencies except to third harmonic frequencies were damped by stack narrow flow channels.

Finally, the distribution of sound pressure in pipe is shown in Fig. 4. The engine room noise was used as sound source. The plotted value was sound pressure at component only $150 \mathrm{~Hz}$. When input sound have the third harmonics mode frequency at $150 \mathrm{~Hz}$, it was found that the stack position was located near the point of antinode in pressure amplitude. In the case of standingwave, the position of antinode in pressure amplitude become the position of node in velocity amplitude.Thus, the maximum value of pressure amplitude and minimum value of velocity amplitudes were obtained at the end of stack. In addition, the acoustic loss is increased with velocity amplitude in the stack. By setting the stack at one-third position in the pipe, the acoustic loss of third harmonic frequency (it was for $150 \mathrm{~Hz}$ in this paper) was less than other frequency by low value of the velocity amplitude. The acsound pressure amplification by thermoacoustic effects was found by installing stack at pressure antiode.

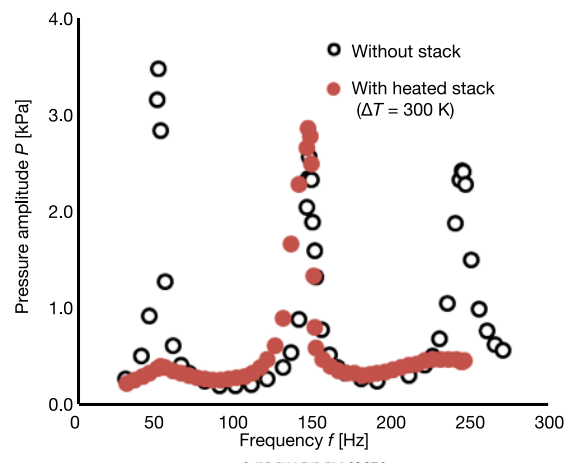

Fig. 2. Resonance curve of pressure amplitude at closed end. 


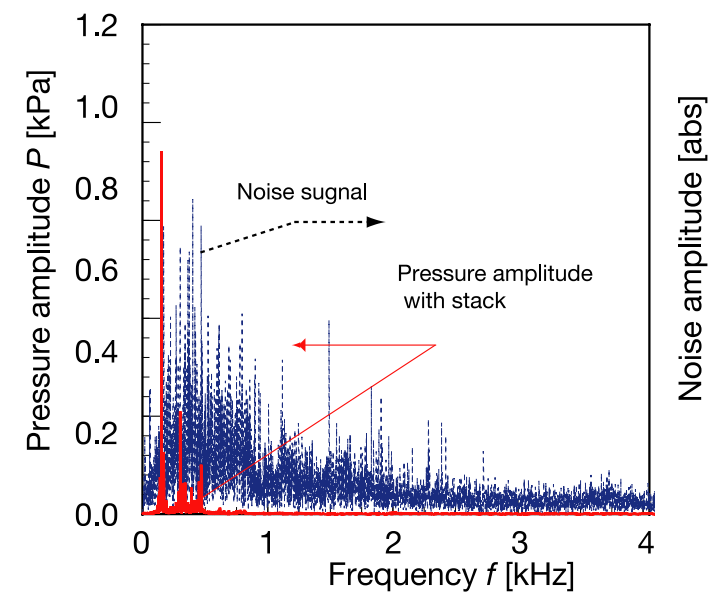

Fig. 3. Consists of sound noise in engine room in WAKASHIOMARU and pressure simplification utilizing stack.

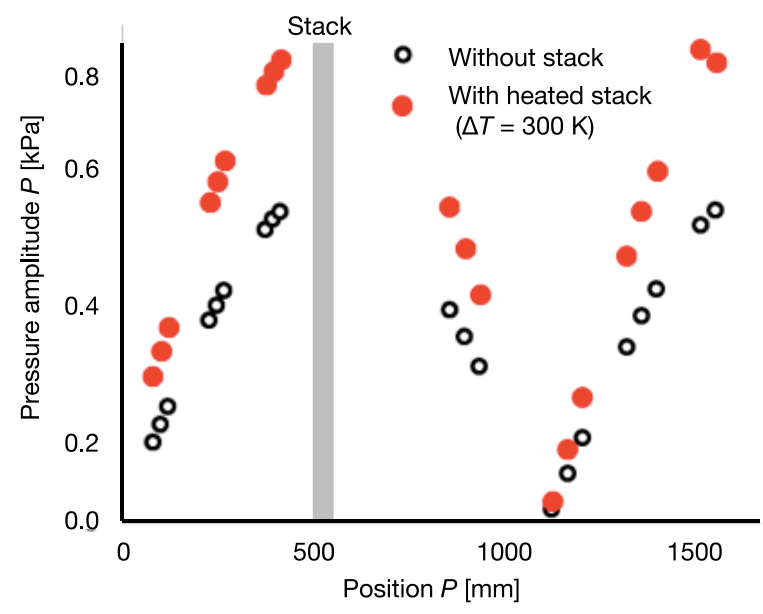

Fig. 4. Sound pressure distribution in pipe.

\section{Conclusion}

In order to utilizing noise energy for thermoacoustic cooler, we demonstrated the sound amplification in a pipe in which stack to temperature gradient was installed. The results showed the amplification of specified frequency and the attenuation of other frequency of sound could be achieved by installing the mesh stack at the pressure amplitude antinode in the pipe.

\section{References}

1. T. Yazaki, A. Iwat, T. Maekawa, and A. Tominaga, A., Phys. Rev. Lett., 81, pp. 3128, (1998).

2. S. Backhause, and G. W. Swift, G "A thermoacoustic Stirling heat engine," Nature, 399, pp. 335-338, (1999).
3. Y. Ueda, T. Biwa, and U. Mizutani, J. Acoust. Soc. Am., 115, 1134, (2004).

4. S. Sakamoto, and Y. Watanabe, Ultrasonics, 42, 53, (2004).

5. Y. Nakamura, and K. Kobayashi K. Proc. of the $3 r d$ International Forum on Heat Transfer, paper No.IFHT2012-202, (2012).

6. S. Hasegawa, T. Yamaguchi, and Y. Oshinoya, Applied Thermal Engineering., 58, 394, (2013).

7. Y. Ueda, and C. Kato, J. Acoust. Soc. Am., 124, 851, (2008).

8. S. Nagata, and Y. Ueda, TEION KOGAKU, 43, 561, (2008).

9. P. C. Ceperley, J. Acoust. Soc. Am., 66, 1508 (1979).

10. T. Biwa, M. Kozuka, and T. Yazaki, Phys. Rev. E, 69, 066304, (2004).

11. T. Biwa, and R. Komatsu J. Acoust. Soc. Am., 129, 132, (2011). 